$09 ; 15$

\title{
Новый метод селективной лазерной
}

\section{наплавки}

(C) Ю.А. Чивель

MerPhotonics, Saint Etienne, France

E-mail: yuri-chivel@mail.ru

Поступило в Редакцию 25 июля 2018 г.

Разработан новый метод селективной лазерной наплавки с использованием конических лазерных пучков. Первоначальный круглый лазерный луч делится на два и более кольцевых пучков с регулируемым распределением мощности лазера по кольцевым пучкам. Кольцевые пучки преобразуются в конические пучки, которые сфокусированы отдельно на поверхности и на различные области наносимого материала для их эффективного нагрева. Ввод энергии лазера в поток порошка очень эффективен, потому что обеспечивается полное равномерное поглощение энергии лазера в плотном потоке порошка $\left(10^{4}-10^{6} 1 / \mathrm{cm}^{3}\right)$. При осаждении проволоки нагрев подложки уменьшает остаточные напряжения из-за градиентов температуры и компенсирует потери тепла из зоны осаждения теплопроводностью. Значительно уменьшается требуемая плотность мощности для формирования металлургического контакта.

DOI: $10.21883 /$ PJTF.2018.22.46928.17472

Эффективность применения конических пучков [1] в лазерной наплавке была впервые показана в [2,3]. Однако внедрение этой инновационной оптической технологии заняло около десяти лет.

В последнее время конические лазерные пучки стали внедряться в технологию лазерной наплавки с применением проволоки [4]. Однако в разработанных устройствах используется один конический пучок, который не обеспечивает оптимального нагрева проволоки и поверхности подложки и требует высокого энергопотребления. Кроме того, предлагаемые оптические системы неэффективны при использовании порошка [5]. С целью устранения указанных недостатков предлагается концепция раздельного нагрева наносимого материала и подложки, на которую он наносится. 
Для раздельного нагрева осаждаемого материала и поверхности разработан метод селективной лазерной наплавки с использованием нескольких конических лазерных пучков [6]. Первоначальный круглый лазерный луч разделяют на несколько кольцевых пучков с регулируемым распределением мощности лазера по кольцевым пучкам. Кольцевые пучки преобразуются в конические пучки, которые фокусируются раздельно на поверхности подложки и на осаждаемом материале (струя порошка, проволока, струя суспензии) для нагрева. Фокусы конических пучков расположены вдоль оптической оси, вдоль которой подается осаждаемый материал.

Для реализации метода разработан ряд устройств.

Устройство работает следующим образом (рис. $1, a$ ). Луч лазера 1 после прохождения через регулируемый расширитель пучка преобразуется в два кольцевых пучка. Один из пучков преобразуется линзой 7 в конический и фокусируется на поверхности 10 в пятно облучения. Наносимый материал подается через трубку 6 в виде потока 9 порошка или проволоки. Другой конический пучок фокусируется с помощью конических зеркал 8 в заданные области потока 9 для нагрева этой области.

Была разработана специальная оптическая система для формирования серии кольцевых лазерных пучков с регулируемым распределением энергии лазера по кольцевым пучкам.

Используя экспандер с регулируемым увеличением и изменяя размеры пучка на выходе, можно перераспределить мощность лазера между кольцевыми пучками и тем самым регулировать нагрев поверхности и потока материала.

В разработанном методе [6] введение энергии лазера в поток порошка более эффективно, так как нагрев малых частиц в стационарном режиме работы и полное равномерное поглощение энергии лазерного луча возможны в плотном потоке порошка. При инжекции порошка структура пучка будет изменена посредством поглощения и рассеяния лазерного излучения. Рассмотрены изменения амплитуды пучка с учетом ослабления лазерного излучения в порошковом слое. Расчеты проводятся для сферических частиц Ті диаметром $d=30 \mu \mathrm{m}$ при объемной плотности $N=10^{6} \mathrm{~cm}^{-3}$. Радиус пучка принимает значение $R=250 \mu \mathrm{m}$. Для когерентной составляющей излучения можно

Письма в ЖТФ, 2018, том 44, вып. 22 


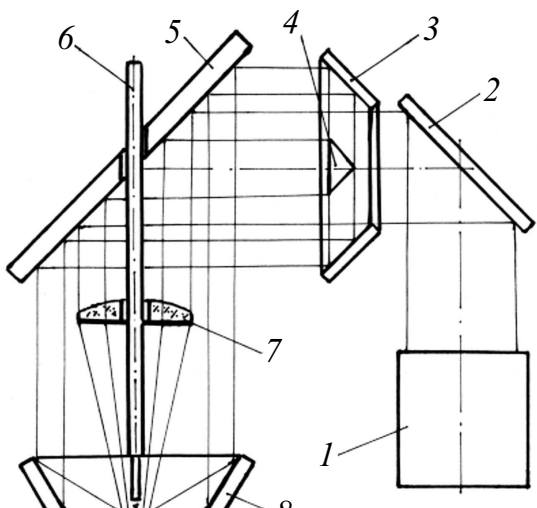

$a$
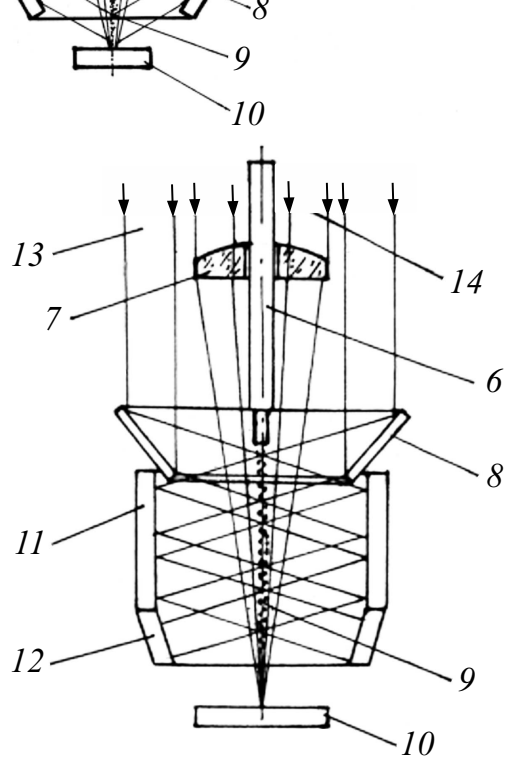

Рис. 1. Оптическая схема устройства лазерной наплавки $(a)$ и многопроходная схема $(b) .1$ - лазер; 2, 5 - поворотные зеркала; $3,4,8,12$ - конические зеркала; 6 - трубка подачи наносимого материала; 7 - линза; 9 - наносимый материал; 10 - подложка; 11 - цилиндрическое зеркало; 13, 14 - кольцевые лазерные пучки. 
использовать экспоненциальную связь между коэффициентом затухания и толщиной слоя [7]:

$$
T_{c}=\exp (-Q \eta),
$$

где $\eta=3 R n_{1} / 2 d-$ коэффициент перекрытия слоя, $Q-$ эффективный коэффициент затухания, $n_{1}=0.74(d / \delta)^{3}$ - безразмерная объемная плотность частиц $(\delta=2 d-$ среднее расстояние между частицами).

Для слоя из оптически жестких больших частиц $(\pi d / \lambda>1$, $(\pi d / \lambda)(n-1) \gg 1)$ эффективный коэффициент затухания можно записать следующим образом:

$$
Q=1 / 1.5 n_{1} \ln \left[1+1.5 n_{1} Q_{i} \exp \left(1.5 n_{1} Q_{i}\right)\right],
$$

пропускание потока порошка имеет вид

$$
T_{c}=\exp [-\sigma(R-r)],
$$

а распределение интенсивности лазерного излучения в порошковом потоке [8]:

$$
q=q_{0}(R / r)\left[e^{-\sigma(R-r)}+e^{-\sigma(R+r)}\right],
$$

где $(R-r)$ - глубина проникновения лазерного излучения в поток порошка, $\sigma$-коэффициент затухания, $Q_{i}-$ коэффициент затухания изолированной частицы. Расчетное значение коэффициента затухания для заданного потока порошка равно $\sigma=45 \mathrm{~cm}^{-1}$, а пропускание когерентной составляющей $T_{c}=0.1$ на диаметре пучка.

Рассеянное лазерное излучение эффективно поглощается частицами при многократном рассеянии. Ввиду достаточно большого диаметра частиц в нашем примере коэффициент пропускания диффузной составляющей также близок к $T_{d}=0.1[9]$.

Исследован нагрев потока порошка. Поток вводится в фокальную область конического пучка длиной $5 \mathrm{~mm}$ со скоростью несколько метров в секунду. Динамика нагрева и испарения сферических частиц описывается уравнениями

$$
\begin{gathered}
(4 / 3) \pi r^{3} \rho C(D t / d t)=\pi r^{2} q Q_{a}-E-L G, \\
d r / d t=-G / 4 \pi r^{2} \rho
\end{gathered}
$$

где $r$ - радиус частицы, $\rho$ - плотность материала частицы, $C-$ удельная теплоемкость, $q-$ интенсивность лазерного излучения,

Письма в ЖТФ, 2018, том 44, вып. 22 
$Q_{a}$ - коэффициент поглощения частицы, $E-$ поток энергии с поверхности частицы, $L-$ удельная теплота испарения, $G$ - массовый расход с поверхности.

На начальной стадии нагрева частиц потери на испарение и теплопроводность малы и температурная динамика может быть записана в виде

$$
T=3 q t Q_{a} / 4 C r .
$$

Согласно расчетам, при длительности пребывания частиц в области нагрева $\sim 10^{-2} \mathrm{~s}$ требуемая мощность лазера для нагрева до $1500 \mathrm{~K}$ не превышает $\sim 200-300 \mathrm{~W}$.

При низкой плотности частиц в потоке порошка эффективность поглощения лазерного излучения резко падает при однократном прохождении излучения через поток. Для максимального использования энергии лазерного излучения разработана многопроходная оптическая схема [6] с использованием цилиндрического 11 и конического 12 зеркал (рис. 1, b). Во-первых, нагрев потока увеличивается, а во-вторых, поглощение лазерного излучения возрастает до полного поглощения при возвращении когерентной части лазерного излучения в лазерный резонатор зеркалом 12 .

Лазерная проволочная наплавка - процесс, который имеет много преимуществ по сравнению с порошковой.

Обычно применяется подача проволоки как сбоку, так и коаксиально при использовании конического пучка [4]. При таком подходе для разогрева как подложки, так и проволоки используются пятна фокусировки миллиметрового размера и мощности лазерного излучения на уровне нескольких киловатт.

При применении двух конических пучков (рис. 2,a) для раздельного нагрева подложки и проволоки достигается оптимальный нагрев проволоки и поверхности подложки.

Для движущегося источника при поверхностном поглощении излучения распределение температуры по поверхности $(\mathrm{z}=0)$ в движущейся системе координат $X Y$ имеет вид [10]:

$$
T(x, y)=\left[A q r^{2} / 2 \pi k\left(x^{2}+y^{2}\right)\right] \exp \left\{\left[-V\left(x+\left(x^{2}+y^{2}\right)^{1 / 2}\right)\right] / 2 a\right\},
$$

где $V$ - скорость, $r$ - радиус фокального пятна, $q-$ плотность мощности, $A-$ коэффициент поглощения, $a-$ коэффициент температуропроводности, $k$ - коэффициент теплопроводности.

Письма в ЖТФ, 2018, том 44, вып. 22 


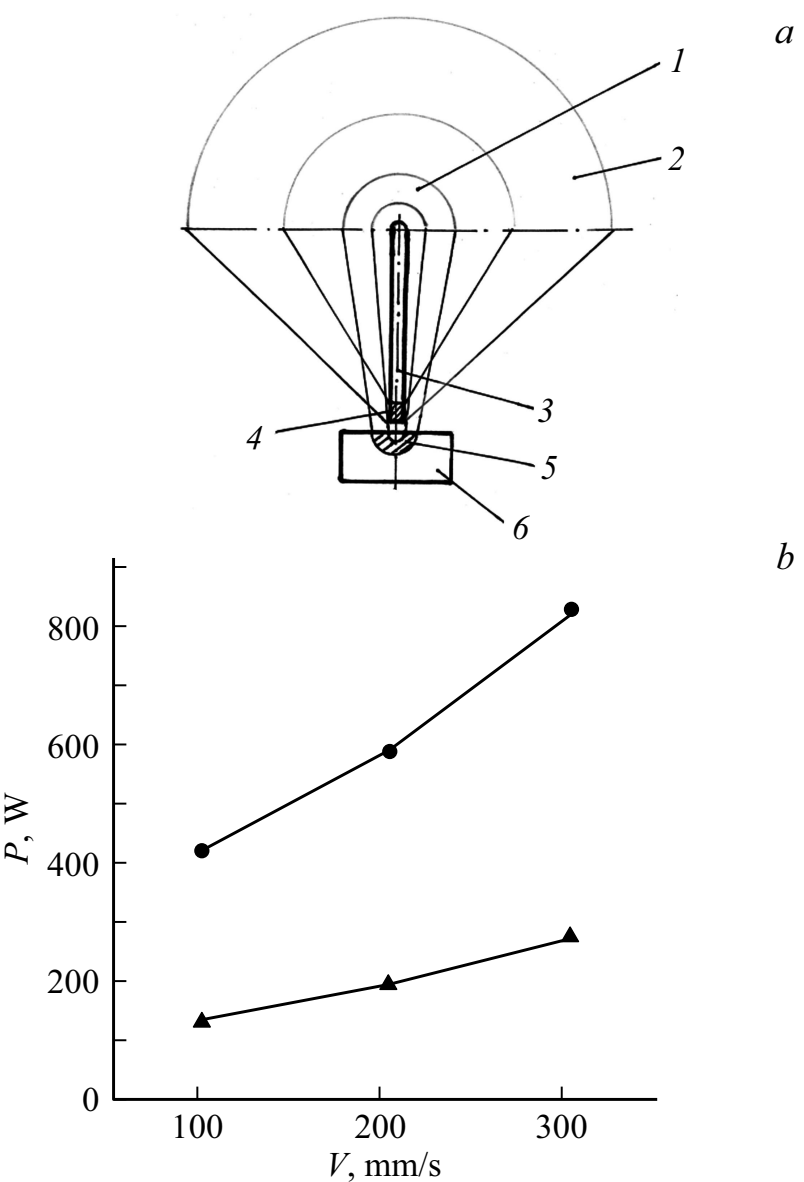

$a$

Pис. 2. $a-$ схема лазерной проволочной наплавки: $1,2-$ конические пучки, 3 - наносимый материал (проволока), 4 - фокальное пятно на проволоке, 5 - фокальное пятно на подложке, 6 - подложка. $b-$ зависимость мощности излучения, достаточной для оплавления поверхности детали (верхняя кривая) и поверхности оконечности проволоки (нижняя кривая), от скорости нанесения.

Существуют простые решения для быстрого и медленного движения пятна нагрева [10]. Критерием выбора решения является соотношение

Письма в ЖТФ, 2018, том 44, вып. 22 

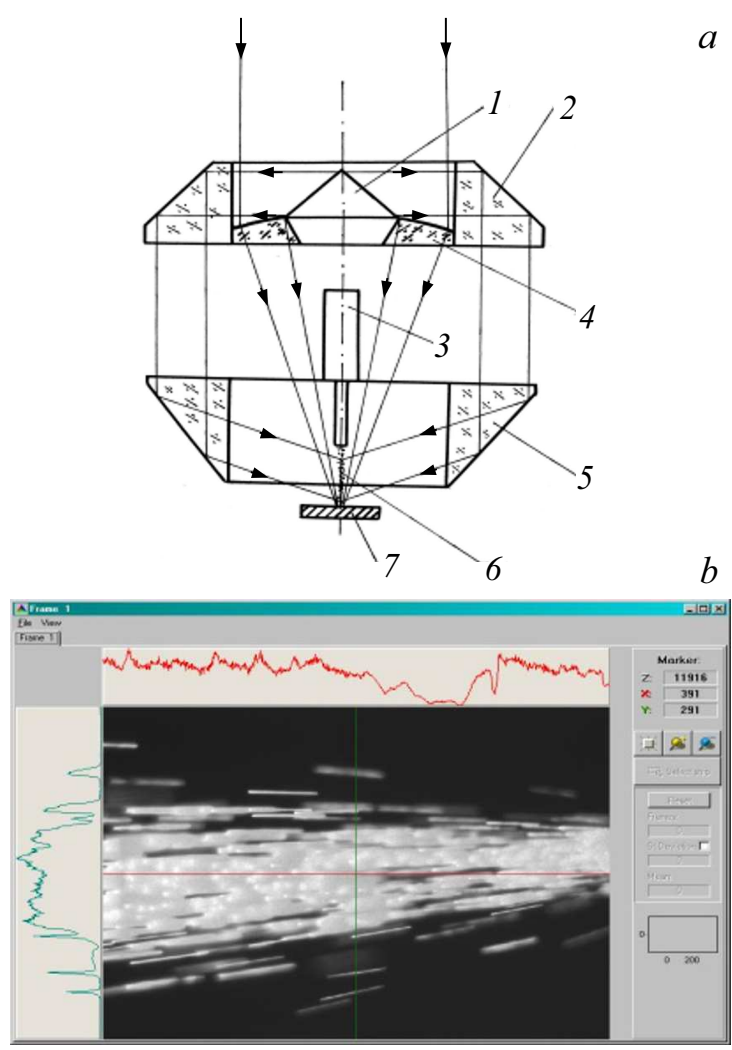

Рис. 3. $a-$ оптическая схема модуля наплавки: 1 - коническое зеркало; 2, 5 - конические призмы; 3 - дозатор порошка; 4 - линза; 6 - поток порошка; 7 - подложка. $b$ - видеоизображение нагрева плотного потока порошка лазерным излучением.

времени экспозиции $t_{e}$ и времени теплопроводности $t_{c}$

$$
t_{e}=2 r / V, \quad t_{c}=r^{2} / a .
$$

Если $t_{c}>t_{e}, V>2 a / r$, то реализуется режим быстрого движения и распределение температуры на поверхности имеет вид

$$
T(0)=2 A q(2 a r)^{1 / 2} / \pi^{3 / 2} k V^{1 / 2} .
$$

Письма в ЖТФ, 2018, том 44, вып. 22 
Проведены расчеты процесса наплавки стальной проволоки диаметром $100 \mu \mathrm{m}$ на стальную подложку. Диаметр пятна фокусировки на подложке составляет $200 \mu \mathrm{m}$, а на проволоке - $80 \mu \mathrm{m}$ (рис. 2, a). Расчеты проводились для режима быстрого нагрева. Результаты показаны на рис. $2, b$. Необходимые мощности намного ниже, чем в стандартных режимах проволочной наплавки при использовании одного лазерного луча.

Использование коаксиальной лазерной наплавки с несколькими коническими пучками позволяет обеспечить меньшую скорость взаимного разбавления, чем в случае других процессов наплавки, при высокой скорости движения и с минимальным вводом тепла.

Разработана и изготовлена экспериментальная система лазерной наплавки на конических пучках (рис. 3). Использованная оптическая схема представлена на рис. $3, a$.

Исследована возможность нагрева плотного потока порошка. $\mathrm{CW}$-диодный лазер мощностью $200 \mathrm{~W}$ был оптически связан с регулируемым расширителем пучка.

Сферический порошок INOX с размером частиц 15-30 $\mu$ m вводился в фокальную область конического пучка длиной $8 \mathrm{~mm}$ через трубку диаметром $0.8 \mathrm{~mm}$ с использованием устройства подачи порошка со скоростью $\sim 2 \mathrm{~m} / \mathrm{s}$. Согласно расчету, плотность частиц в фокальной области составляла около $10^{5} \mathrm{~cm}^{-3}$. Такая плотность на несколько порядков выше, чем в обычно используемых системах лазерной наплавки.

На рис. $3, b$ представлен порошковый поток под действием лазерного излучения, зарегистрированный CMOS-камерой. Наблюдается равномерный прогрев струи.

Таким образом, для раздельного нагрева осаждаемого материала и поверхности разработаны новый метод и система лазерной наплавки с использованием нескольких конических лазерных пучков.

Пространственно-независимый и разделенный нагрев поверхности и потока осаждаемого материала обеспечивает оптимальные условия термического процесса, значительно снижает энергозатраты и повышает точность и качество наплавки.

\section{Список литературы}

[1] McLeod J. // J. Opt. Soc. Am. 1954. V. 44. P. 592-596.

[2] Чивель Ю.А. // Письма в ЖТФ. 2005. Т. 31. В. 1. С. 3-8.

[3] Chivel Yu. Patent BY N 6931. 10.11.2004.

Письма в ЖТФ, 2018, том 44, вып. 22 
[4] Pitch O., Stollenwerk J., Kogel-Hollacher M., Traub M. // Adv. Opt. Tech. 2012. V. 1. P. $397-402$.

[5] Kuznetsov A., Jeremen A., Levy G., Kruth J.-P. // Phys. Procedia. 2016. V. 83. P. 647-656.

[6] Chivel Yu. Patent RU N 2580180. US Pat. Appl. 20170312856.

[7] Ivanov A.P., Loiko V.A., Dik V.P. The propagation of light in snug-packing dispersed medium. Minsk, 1988. $320 \mathrm{p}$.

[8] Schafer E.P. // Appl. Phys. 1986. V. 39. P. 1-8.

[9] Науменко Е.А., Чивель Ю.А. // Изв. вузов. Приборостроение. 2006. № 9. C. $55-58$.

[10] Либенсон М.Н., Яковлев Е.Б., Шандыбина Г.Д. Взаимодействие лазерного излучения с веществом. СПб.: ИТМО, 2014. Ч. 2. 181 с. 